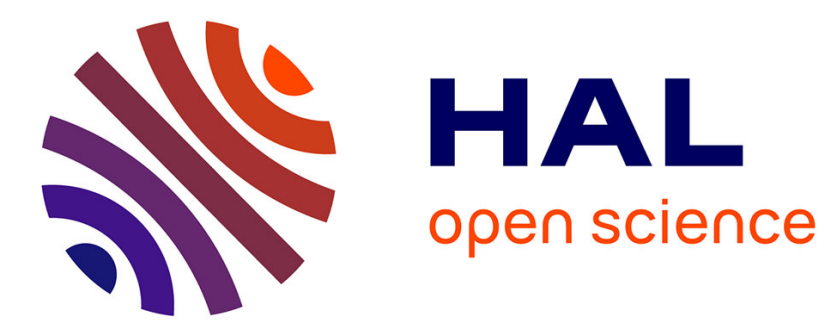

\title{
Les étalons de rayonnement dans l'ultraviolet
}

P. Fieffe-Prevost, J.P. Watteau, F. Wuilleumier

\section{To cite this version:}

P. Fieffe-Prevost, J.P. Watteau, F. Wuilleumier. Les étalons de rayonnement dans l'ultraviolet. Revue de Physique Appliquée, 1977, 12 (6), pp.913-925. 10.1051/rphysap:01977001206091300 . jpa00244261

\section{HAL Id: jpa-00244261 https://hal.science/jpa-00244261}

Submitted on 1 Jan 1977

HAL is a multi-disciplinary open access archive for the deposit and dissemination of scientific research documents, whether they are published or not. The documents may come from teaching and research institutions in France or abroad, or from public or private research centers.
L'archive ouverte pluridisciplinaire HAL, est destinée au dépôt et à la diffusion de documents scientifiques de niveau recherche, publiés ou non, émanant des établissements d'enseignement et de recherche français ou étrangers, des laboratoires publics ou privés. 


\title{
REVUE DE PHYSIQUE APPLIQUÉE
}

\author{
Classification \\ Physics Abstracts \\ 0.643

\section{LES ÉTALONS DE RAYONNEMENT DANS L'ULTRAVIOLET}

\author{
P. FIEFFE-PREVOST, J. P. WATTEAU \\ Institut National de Métrologie, Conservatoire National des Arts \& Métiers \\ 292, rue Saint-Martin, 75003 Paris, France \\ et \\ F. WUILLEUMIER \\ E. R. no 184 du C. N. R. S., Université Paris-Sud, 91405 Orsay Cedex, France \\ (Reçu le 28 janvier 1977, accepté le 11 mars 1977)
}

\begin{abstract}
Résumé. - Après avoir défini les caractéristiques d'une source étalon, nous décrivons les principales sources utilisées. Pour chaque source nous précisons la méthode d'excitation, la loi de rayonnement et le domaine d'application ainsi que la précision. Nous expliquons tout d'abord les sources émettant un spectre de raies (méthode des coïncidences, excitation par impact électronique, rapport de branchement, raies saturées) puis les sources émettant un spectre continu (arc hydrogène, rayonnement synchrotron, rayonnement Cerenkov). Enfin, nous mentionnons les sources de transfert les plus utilisées.

Abstract. - We first describe the essential properties of a standard source. We then review the main sources used for spectral measurements from few Angstroms to the visible. For each source we point out the excitation mode, the radiation law, the range of application and the accuracy. We have separated the line sources (coincidence method, excitation by electronic impact, branching ratio, Boldt Method) from the continuum sources (hydrogen arc, synchroton radiation, Cerenkov radiation). Lastly we mention the transfer standards.
\end{abstract}

1. Introduction. - Depuis quelques années nous assistons à une croissance importante des recherches dans le domaine ultraviolet. Cette croissance est due à l'amélioration des techniques (techniques du vide, source de rayonnement, détection) et au développement de la physique spatiale, de la physique des plasmas et des expériences sur la fusion thermonucléaire contrôlée ou encore de l'étude des interactions de l'homme avec son milieu environnant (pollution, dégradation de matériaux sous l'effet du rayonnement U. V., etc...). Ces recherches nécessitent la mesure de flux énergétiques rayonnants, soit pour leur interprétation, soit pour établir des comparaisons entre laboratoires. Pour effectuer cette mesure deux solutions s'offrent à l'expérimentateur. La première consiste à étalonner le dispositif optique, c'est-à-dire à connaître sa fonction de transfert, et à utiliser comme récepteur un détecteur absolu (photodiode, chambre à ionisation, etc...). La deuxième solution, plus simple, est de comparer, à travers le même dispositif optique, la source étudiée et une source étalon, source dont le rayonnement est connu. Le choix entre ces deux solutions dépend principalement de l'intervalle spectral étudié et de l'ordre de grandeur des puissances à mesurer. Nous avons indiqué dans le tableau I les principaux types de sources pouvant être utilisées comme étalon. Elles se divisent en deux catégories : les sources donnant un spectre de raies et celles donnant un spectre continu. Après avoir rappelé les caractéristiques d'une source étalon, nous décrirons les méthodes les plus employées sur lesquelles s'appuie le calcul de leur rayonnement.

2. Caractéristique d'une source étalon. - Une source étalon est une source de rayonnement dont la puissance émise est calculable en fonction de grandeurs physiques mesurables. Par exemple, dans le domaine visible on emploie la lampe à ruban de tungstène ou l'arc au carbone qui tous deux rayonnent sensiblement comme un corps noir. On calcule alors leur luminance spectrale énergétique $L_{\lambda}$ à l'aide de la loi de Planck, en fonction de la longueur d'onde et de la température. La température de ces sources étant de l'ordre de $3000 \mathrm{~K}$, d'après la loi de Wien :

$$
\lambda_{\mathrm{M}} T=2885 \mu \mathrm{m} . \mathrm{K}
$$

$T$ : température $(\mathrm{K})$

$\lambda_{\mathrm{M}}$ : longueur d'onde du maximum de $L_{\lambda}(\mu \mathrm{m})$. 


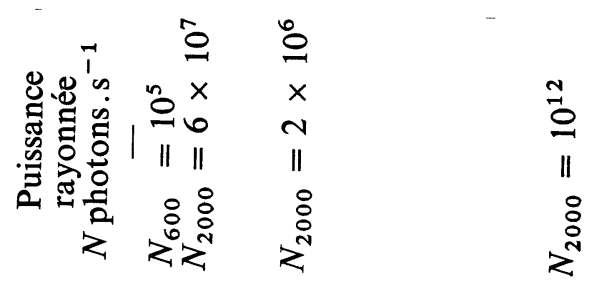

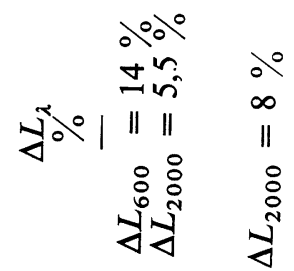

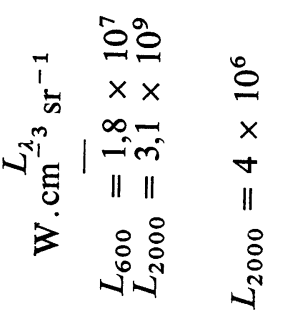

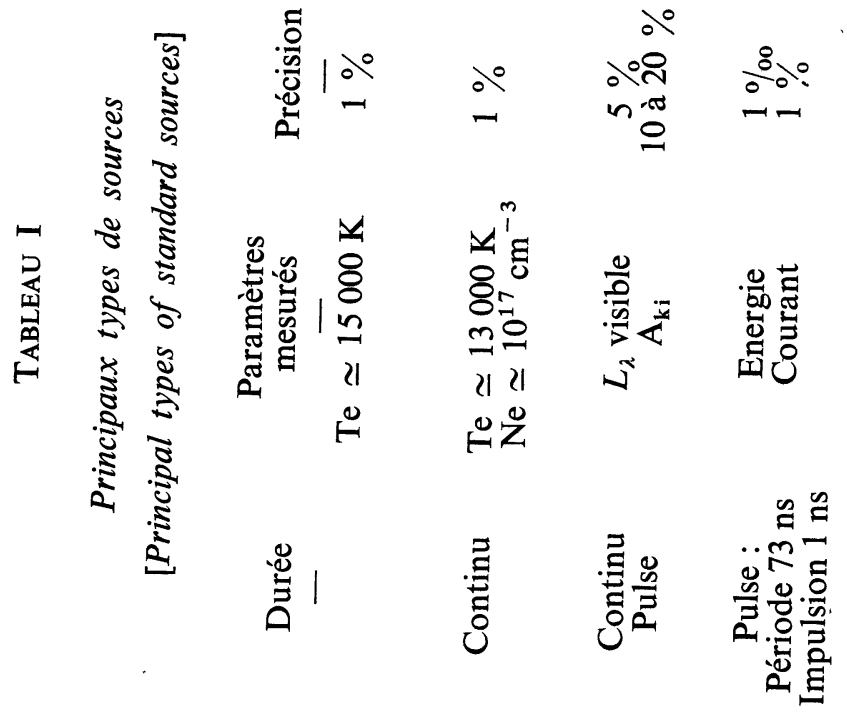

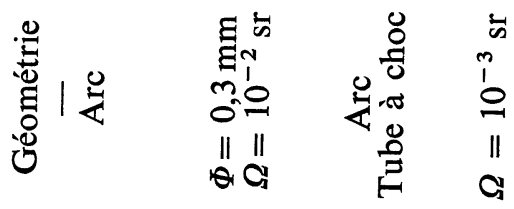

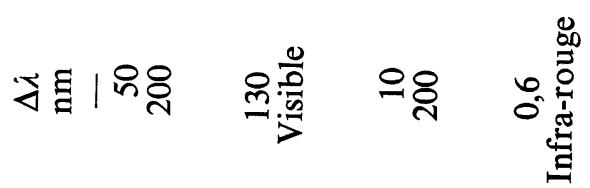

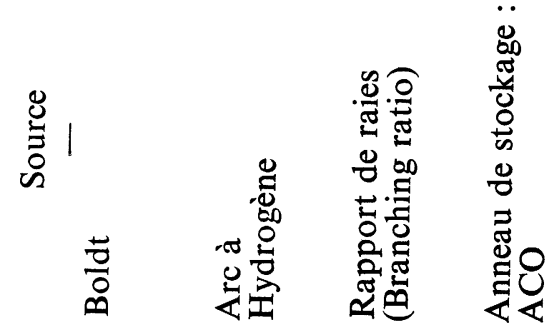


Leur émission se situe principalement dans le proche infrarouge (Fig. 1). Elles ne sont donc plus utilisables en dessous de $300 \mathrm{~nm}$. Aussi, il est nécessaire de disposer de sources émettant davantage dans

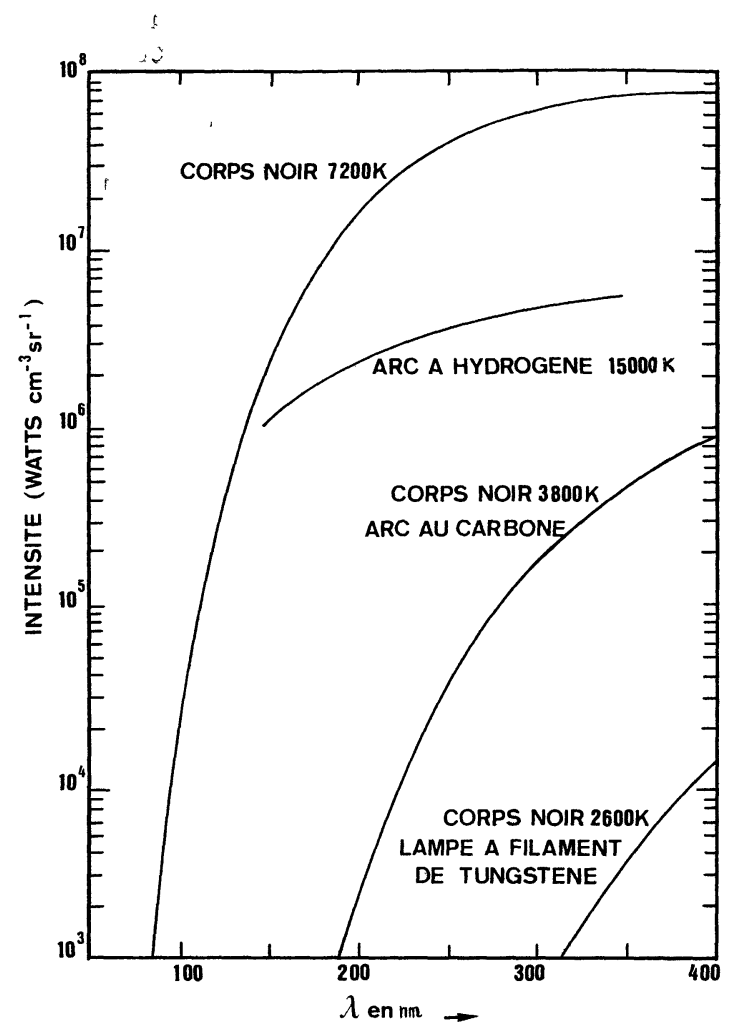

FIG. 1. - Luminances comparées des différentes sources.

[Spectral radiance of standard sources.]

l'ultraviolet. Ces sources doivent être stables et reproductibles de manière à ce que la précision des mesures soit la meilleure possible. D'autre part, il faut que le phénomène à l'origine de leur émission soit bien connu afin que le rayonnement calculé en fonction des grandeurs physiques soit le plus proche du rayonnement réel pour réduire les erreurs systématiques. Les sources fonctionnent soit en régime pulsé soit en régime continu.

3. Sources étalon émettant un spectre de raies. Nous allons tout d'abord décrire quelques méthodes basées sur la mesure du rapport d'intensités de raies. Les deux premières méthodes utilisent comme moyen d'excitation l'impact par faisceau électronique.

3.1 MÉTHODE DES COÏNCIDENCES. - La source est constituée par un gaz excité par un faisceau d'électrons. Considérons, par exemple, les collisions entre des molécules d'hydrogène et les électrons du faisceau. La plupart de ces collisions ont pour effet non seulement de dissocier les molécules mais également d'exciter les atomes ainsi formés. Quelques-uns des atomes excités dans l'état $n=3$ vont se désexciter en passant successivement dans l'état $n=2$ avec l'émission d'un photon à $656,3 \mathrm{~nm}$ et dans l'état $n=1$ émettant un photon à $121,6 \mathrm{~nm}$. Si les deux photons sont détectés en coïncidence le rapport entre le nombre de coïncidences et le nombre de photons détectés à $656,3 \mathrm{~nm}$ donne l'efficacité de la source à $121,6 \mathrm{~nm}$. Ayant mesuré le flux émis à $656,3 \mathrm{~nm}$ par comparaison avec une lampe à filament de tungstène, on en déduit le flux 121,6 nm [1]. La précision de la méthode est de l'ordre de $20 \%$.

3.2 MÉThOde De CAlibration POUR LA SPECTROMÉTRIE U. V. BASÉE SUR L'EXCITATION PAR IMPACT ÉleCTroniQue. - Comme son nom l'indique cette méthode est spécialement adaptée à la mesure des interactions par bombardement électronique. Elle est basée sur le calcul de la section efficace d'émission de raies de résonance des gaz rares [2,3]. Les niveaux supérieurs sont excités par les électrons rapides. La mesure de l'intensité relative d'une raie en fonction de l'énergie du faisceau électronique permet alors de déterminer de façon absolue l'efficacité quantique d'un spectromètre à condition que la force d'oscillateur de la transition soit connue. L'intensité lumineuse des raies de résonance de $\mathrm{He}, \mathrm{Ne}, \mathrm{Ar}, \mathrm{Kr}$ a ainsi été mesurée pour des énergies comprises entre 100 et $2000 \mathrm{eV}$. Le courant électronique est de l'ordre de $200 \mu \mathrm{A}$ et la pression du gaz dans la chambre varie entre $10^{-7}$ et $10^{-6} \mathrm{~Pa}$. Il faut, en général, tenir compte des phénomènes de cascades qui peuplent le niveau de résonance et de l'absorption des raies de résonance qui n'est pas négligeable malgré les faibles pressions du gaz [4]. Enfin, la lumière émise est polarisée du fait du caractère unidirectionnel du faisceau excitant le gaz. Cette méthode est applicable entre 50 et $120 \mathrm{~nm}$. L'erreur expérimentale sur la détermination de l'efficacité quantique est de l'ordre de $20 \%$.

3.3 MÉTHODE DU RAPPORT DE BRANCHEMENT (BRANCHING RATIO) [5]. - La méthode consiste à mesurer le rapport des intensités de deux raies situées l'une dans le visible, l'autre dans l'ultraviolet, les deux raies ayant le même niveau supérieur $k$. Les coefficients d'émission dans le visible, $\varepsilon_{\mathrm{vis}}$ et dans $l^{\prime}$ U. V., $\varepsilon_{\mathrm{U} . v .}$, ont pour expression :

$$
\begin{aligned}
\varepsilon_{\mathrm{vis}} & =\frac{h c}{4 \pi \lambda_{\mathrm{vis}}} \cdot A_{\mathrm{ik}} N_{\mathrm{k}} \\
\varepsilon_{\mathrm{U} . \mathrm{v} .} & =\frac{h c}{4 \pi \lambda_{\mathrm{U} . \mathrm{v} .}} A_{\mathrm{jk}} N_{\mathrm{k}}
\end{aligned}
$$

$h$ : constante de planck

$c$ : vitesse de la lumière dans le vide

$A_{\mathrm{ik}}$ : probabilité de transition du niveau $\mathrm{k}$ vers le niveau i

$N_{\mathbf{k}}$ : nombre d'atomes excités dans l'état $\mathbf{k}$ par unité de volume.

Le rapport des luminances s'écrit :

$$
\frac{L_{\mathrm{U} . \mathrm{v}}}{L_{\mathrm{vis}}}=\frac{\varepsilon_{\mathrm{U} . \mathrm{v} .} l}{\varepsilon_{\mathrm{vis}} l}=\frac{\lambda_{\mathrm{vis}}}{\lambda_{\mathrm{U} . \mathrm{v}}} \cdot \frac{A_{\mathrm{jk}}}{A_{\mathrm{ik}}}
$$

$l$ longueur de la source suivant le chemin optique. 
On mesure la luminance de la raie visible à l'aide d'une lampe à ruban de tungstène, et connaissant les longueurs d'onde et les probabilités de transition des raies, on en déduit la luminance de la raie $U$. V.

Comme dans les méthodes précédentes, il est très important que les phénomènes d'absorption soient négligeables. En outre, si le niveau supérieur présente une structure fine il faut faire la moyenne des probabilités de transition des sous-niveaux en tenant compte de leur poids statistique.

Pour les systèmes atomiques cette méthode ne s'applique qu'à quelques couples de raies (voir Tableau II). Par contre, la méthode a été étendue aux systèmes moléculaires tels que $\mathrm{H}_{2}, \mathrm{~N}_{2}, \mathrm{CO}$ ou NO [6]. Pour deux états électroniques $\mathrm{v}^{\prime}$ et $\mathrm{v}^{\prime \prime}$ de la molécule, correspondant à une transition électrique dipolaire permise, la probabilité d'une telle transition a pour expression :

$$
A_{\mathrm{v}^{\prime} \mathrm{v}^{\prime \prime}}=\frac{64 \pi^{4} q_{\mathrm{v}^{\prime} \mathrm{v}^{\prime \prime}}}{3 h \lambda_{\mathrm{v}^{\prime} \mathrm{v}^{\prime \prime}}^{3}} R_{\mathrm{e}}^{2}\left(\mathrm{v}^{\prime} \mathrm{v}^{\prime \prime}\right),
$$

$q_{\mathrm{v}^{\prime} \mathrm{v}^{\prime \prime}} \quad$ : facteur de Franck et Condon [7]

$\lambda_{\mathrm{v}^{\prime} \mathrm{v}^{\prime \prime}} \quad:$ longueur d'onde de la raie

$R_{\mathrm{e}}\left(\mathrm{v}^{\prime} \mathrm{v}^{\prime \prime}\right)$ : moment électronique de la transition.

\section{TABLEAU II}

Exemple de couples de raies utilisés dans la méthode des rapports de branchement

$\begin{array}{cccc}\text { Gaz } & \lambda_{\text {U.v. }}(\mathrm{nm}) & \lambda_{\text {vis }}(\mathrm{nm}) & \begin{array}{c}A_{\text {jkU.v. }} \\ A_{\text {ikvis }}\end{array} \\ \overline{\mathrm{H}_{\mathrm{I}}} & 102,6 & 656,3 & 1,28 \\ & 97,3 & 486,1 & 1,54 \\ \mathrm{He}_{\text {II }} & 95,0 & 434,1 & 1,60 \\ & 24,3 & 468,6 & 1,44 \\ & 23,7 & 320,3 & 1,82 \\ \mathrm{Li}_{\text {III }} & 121,5 & 468,6 & 0,93 \\ & 108,5 & 320,3 & 1,14 \\ & 10,5 & 449,9 & 1,48 \\ & 48,2 & 449,9 & 0,93\end{array}$

La polarisation du rayonnement émis est en général négligeable car de nombreux niveaux de rotation sont excités dont la moyenne donne un taux de polarisation proche de zéro pour la bande. L'intérêt des bandes moléculaires au point de vue de la calibration d'un système réside dans le fait que les très nombreuses raies permettent un étalonnage quasi continu. Des mesures de sections efficaces par cette méthode semblent indiquer que les erreurs sont de l'ordre de $25 \%$.

3.4 MéThode des RAIES SATURÉEs [8]. - Cette méthode est fondamentalement différente des précédentes dans son principe car elle est basée sur la réabsorption du rayonnement. Pour un plasma en équilibre thermodynamique local et homogène [9] l'équation de la luminance spectrale énergétique le long du chemin optique s'écrit :

$$
L_{\lambda}=L_{\mathrm{e} \lambda}\left[1-\exp \left(-K_{\lambda} l\right)\right]
$$

où $l$ est la longueur de la source, $K_{\lambda}$ son facteur d'absorption. $L_{\mathrm{e} \lambda}$ est la luminance du corps noir :

$$
L_{\mathrm{e} \lambda}=\frac{\varepsilon_{\lambda}}{K_{\lambda}}=\frac{2 h c^{2}}{\lambda^{5}} \frac{1}{\exp \left(\frac{h c}{\lambda K T}\right)-1}
$$

$K$ : constante de Boltzmann

$\varepsilon_{\lambda}$ : coefficient d'émission.

Si l'épaisseur optique $K_{\lambda} \times l$ est beaucoup plus grande que l'unité, $L_{\lambda} \simeq L_{\mathrm{e} \lambda}$ : la luminance qui ne dépend plus que de la température et de la longueur d'onde, est donnée par la formule (5). La condition $K_{\lambda} l \gg 1$ est assez facilement réalisable pour des raies de résonance dans un plasma à pression voisine de la pression atmosphérique. Une vingtaine de raies de résonance a été dénombrée par Boldt [8] entre 80 et $250 \mathrm{~nm}$.

Expérimentalement on utilise un arc électrique sous pression atmosphérique d'argon dans lequel le gaz, dont les raies constituent les références cherchées, est introduit, à faible débit, au centre de l'arc. La détermination de la température de l'arc s'effectue de la façon suivante :

- Mesure de la densité électronique par élargissement Stark.

- Mesure du rapport des intensités d'une raie d'argon neutre et d'une raie d'argon ionisé. L'équilibre thermodynamique local étant réalisé, ce rapport est proportionnel à la densité électronique et fonction de la température.

Connaissant celui-ci et la densité électronique, on en déduit la température. A partir de l'équation (5) on calcule la luminance spectrale énergétique de la raie référence pour laquelle l'arc est optiquement épais.

Le rayonnement des raies de référence a été comparé par l'intermédiaire d'étalons de transfert (lampe au deutérium), au rayonnement synchrotron [10]: les déviations maximales entre les deux types de mesure sont inférieures à $\pm 3 \%$.

4. Spectre continu. - Pour l'expérimentateur qui désire étalonner son appareillage, il est plus pratique de disposer d'une source émettant un rayonnement continu. Mais ce rayonnement continu est, en général, beaucoup plus difficile d'une part à produire et d'autre part à calculer. Si la première méthode présentée dans ce paragraphe met en jeu des moyens classiques, les deux suivantes découlent des techniques nucléaires.

4.1 Continu DE L'HYdrogène. - C'est le fond continu de l'hydrogène atomique entre 130 et $380 \mathrm{~nm}$ 
qui constitue la source de référence. Le coefficient d'émission du fond continu $\varepsilon_{\lambda}\left(n_{\mathrm{e}}, T\right)$, dû au rayonnement de freinage entre les électrons, les ions et les atomes [11] est calculable en fonction de la densité électronique $n_{\mathrm{e}}$ et de la température $T$, du plasma. Celui-ci étant optiquement mince dans l'intervalle spectral considéré, la luminance spectrale d'une colonne homogène est égale, d'après la formule (4) pour $K_{\lambda} l \ll 1$, à :

$$
L_{\lambda} \simeq \varepsilon_{\lambda}\left(n_{\mathrm{e}}, T\right) . l .
$$

Le plasma est créé par un arc électrique de type Maecker [12] (Fig. 2). L'arc est constitué par une

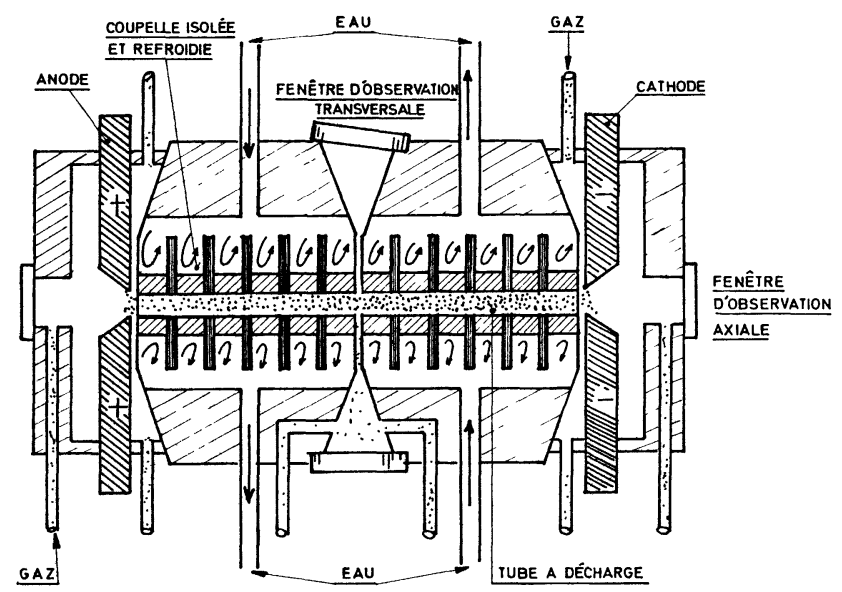

Fig. 2. - Schéma de l'arc électrique.

[The wall stabilized arc.]

série de coupelles en cuivre, empilées et isolées électriquement les unes des autres. Elles sont percées en leur centre d'un trou circulaire qui constitue le tube à décharge. Aux deux extrémités se situent les électrodes alimentées par un générateur de courant stabilisé. La pression du gaz dans le tube à décharge est égale à la pression atmosphérique : de nombreuses arrivées et sorties de gaz permettent de contrôler la stabilité du plasma le long de la colonne. Les électrodes en tungstène et les coupelles sont refroidies le plus souvent par eau (débit de l'ordre de quelques dizaines de litres par minute). L'épaisseur des coupelles est de $2 \mathrm{~mm}$, et le diamètre de la décharge de $3 \mathrm{~mm}$. Pour une longueur de plasma de $30 \mathrm{~mm}$ et une température au centre de $13000 \mathrm{~K}$, une puissance électrique continue de $20 \mathrm{~kW}$ est nécessaire pour alimenter l'arc. Ce type de décharge possède plusieurs avantages : il est robuste, stable et fonctionne en régime continu.

Le plasma étant proche de l'équilibre thermodynamique local les densités des différentes espèces et la température sont reliées entre elles par des lois simples [29]. Il suffit donc de déterminer l'une quelconque de ces grandeurs pour connaître les autres. On effectue, en général, les diagnostics suivants :
- Mesure de la densité électronique par élargissement Stark de la raie $\mathrm{H}_{\beta}$.

- Détermination de la densité d'un niveau excité par mesure de la luminance des raies de la série de Balmer, en particulier $\mathrm{H}_{\beta}$ et $\mathrm{H}_{\gamma}$.

- Mesure de la temperature déduite du rapport de l'intensité spectrale du fond continu et de l'intensité d'une raie.

- Mesure absolue du fond continu dans le domaine visible.

Ces divers diagnostics permettent de déterminer la densité électronique et la température du plasma et par suite la luminance du fond continu (formule 6). Une erreur inférieure à $1 \%$ sur la température entraîne une incertitude sur la luminance du fond continu de l'ordre de $10 \%$ à $200 \mathrm{~nm}$.

Un des intérêts de cette source est d'émettre un fond continu entre $130 \mathrm{~nm}$ et $380 \mathrm{~nm}$ de forte luminance (Fig. 3). En dessous de $140 \mathrm{~nm}$ la connaissance

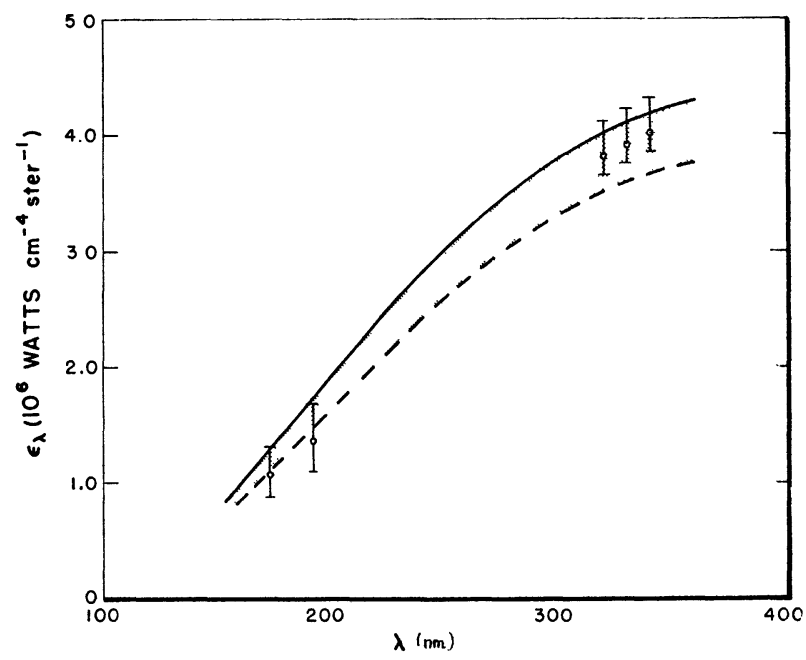

Fig. 3. - Coefficient d'émission de l'hydrogène.

[Emission coefficient of hydrogen.]

$\mathrm{du}$ rayonnement en valeur absolue devient plus imprécise du fait de la contribution des ailes de la raie Lyman $\alpha$. Au-delà de $100 \mathrm{~nm}$ le fond continu n'est plus optiquement mince et n'a pas encore été étudié. Le fond continu ainsi mesuré a été comparé expérimentalement d'une part à l'émission de raies saturées, et d'autre part au rayonnement d'une lampe à ruban de tungstène dans le proche U. V. Sur la figure 3 on a représenté en traits pleins la luminance de l'arc calculée à partir de la température électronique mesurée, et en grisé l'incertitude liée aux problèmes d'alignement. Les points représentent les mesures effectuées d'une part avec la lampe au-dessus de $300 \mathrm{~nm}$ et d'autre part avec les raies de résonance de $C_{I}$ et $N_{I}$ au voisinage de $160 \mathrm{~nm}$ [13]. Une telle source est actuellement opérationnelle à l'Institut National de Métrologie. Une autre de ces sources est en fonctionnement au National Bureau of Stan- 
-dards. Elle utilise les propriétés du maximum d'émission pour une température de l'ordre de $18000 \mathrm{~K}$. L'avantage de cette méthode est de s'affranchir entièrement d'une mesure absolue dans le domaine visible [27].

Des comparaisons sont actuellement en cours entre l'arc hydrogène et le rayonnement synchrotron décrit ci-dessous.

4.2 RAYONNEMENT SYNCHROTRON. - 4.2.1 Caractéristiques générales du rayonnement. - Une particule soumise à une accélération quelconque émet un rayonnement électromagnétique [14]. Dans le cas d'une orbite circulaire (accélération centripète), la puissance totale rayonnée par un électron effectuant une révolution s'écrit :

$$
\Delta E=\frac{4 \pi}{3} \frac{e^{2}}{R} \gamma^{4}
$$

où $R$ est le rayon magnétique de la trajectoire et $\gamma=E / m_{0} c^{2}, E$ étant l'énergie de l'électron. Le rayonnement émis est continu et s'étend depuis le domaine $\mathrm{X}$ ou X-UV (suivant l'énergie de l'électron) jusqu'au domaine de l'infrarouge lointain. La distribution spectrale émise présente un maximum vers les courtes longueurs d'onde du spectre maximum dont la position en longueur d'onde est proportionnel à $R / E^{3}$. A une longueur d'onde de l'ordre de $\lambda_{M} / 4$ il n'y a pratiquement plus de rayonnement utilisable. La décroissance vers les grandes longueurs d'onde est beaucoup plus lente.

Le déplacement relativiste des particules sur leur trajectoire conduit à une focalisation du rayonnement émis suivant la tangente à l'orbite, la divergence du rayonnement étant d'autant plus faible que l'énergie de la particule est plus élevée et le photon émis plus dur. L'électron émettant un tel rayonnement en chaque point de sa trajectoire, la propriété de faible divergence est perdue dans le plan horizontal et le rayonnement émis est distribué à l'intérieur d'une nappe conique centrée sur la trajectoire et présentant, dans le plan vertical, une divergence très faible caractérisée par l'angle d'émission $\psi$ avec le plan de l'orbite (voir Fig. 4).

Le rayonnement émis se divise en deux composantes: l'une polarisée parallèlement au plan de l'orbite, l'autre perpendiculairement. De plus ces deux composantes présentent entre elles une différence de phase de $\pi / 2$ et le rayonnement émis est polarisé elliptiquement. L'intensité relative des deux composantes dépend de l'angle d'émission $\psi$. Pour $\psi=0$, la composante perpendiculaire est nulle et le rayonnement émis est polarisé linéairement à $100 \%$ dans le plan de l'orbite.

Enfin dans les domaines de longueur d'onde intéressants, le rayonnement émis ne présente aucune propriété de cohérence particulière, car la densité des électrons sur la trajectoire est trop faible pour conduire à une corrélation entre particules ou à une émission stimulée.

D'après l'équation (7), la puissance rayonnée est d'autant plus grande que la masse de la particule est plus faible et la théorie prévoit que seuls des électrons (ou des positrons) auront une masse suffisamment faible pour émettre du rayonnement utilisable. Ce rayonnement a donc été observé et utilisé auprès de synchrotrons ou d'anneaux de stockage à électrons.

\subsubsection{Les accélérateurs circulaires d'électrons. -} Il existe deux sortes de machines produisant $\mathrm{du}$ rayonnement synchrotron à savoir les synchrotrons et les anneaux de collisions ou anneaux de stockage, que nous allons comparer brièvement dans ce qui suit. Dans ces machines l'énergie perdue à chaque tour par rayonnement synchrotron est fournie aux électrons par une cavité radiofréquence placée sur une section droite de la chambre à vide.

Les synchrotrons produisent des faisceaux d'électrons de quelques $\mathrm{mA}$, de grande énergie (de quelques centaines de $\mathrm{MeV}$ à quelques $\mathrm{GeV}$ ). Le champ magnétique des aimants dans les sections courbes est en

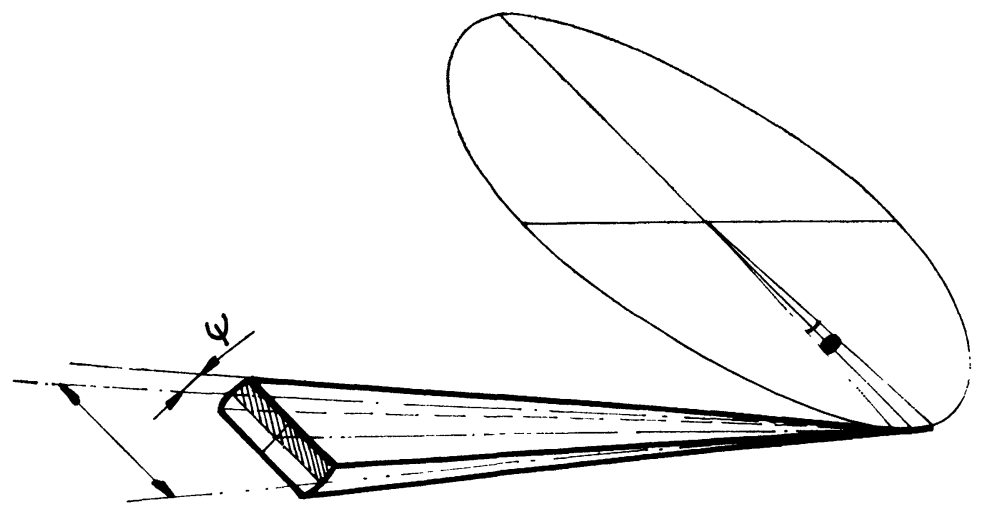

FIG. 4. - Caractéristiques géométriques du rayonnement émis par un électron accéléré circulairement. L'angle d'émission $\psi$ caractérise la divergence verticale du rayonnement.

[Geometric characteristics of the radiation emitted by an electron moving in a circle. $\psi$ represents the vertical divergence.] 
permanence ajusté en fonction de l'énergie des électrons. Un paquet d'électrons injecté dans la machine est accéléré durant un temps qui dure typiquement $10 \mathrm{~ms}$, envoyé sur la cible, puis un nouveau paquet est injecté et ainsi de suite. Durant l'accélération des électrons, il y a un déplacement vers les hautes énergies de la distribution du rayonnement synchrotron émis ; cependant, d'un cycle à l'autre, ce déplacement se reproduit identiquement et la distribution spectrale moyennée sur un cycle est parfaitement constante ; l'intensité émise subit donc une variation périodique programmée rigoureusement. Par contre le nombre d'électrons injectés à chaque cycle peut varier, c'est-àdire que le flux de photons émis fluctue également de façon aléatoire dans le temps. De plus dans un synchrotron, l'orbite suivie par les paquets d'électrons n'est pas parfaitement stable et peut varier, dans le plan vertical, de plusieurs millimètres.

Le rayonnement émis par ces machines présente donc une structure temporelle. Dans chaque paquet d'électrons injectés, les électrons ne sont pas répartis uniformément, car la radiofréquence accélératrice les agglutine par paquets de quelques fractions de millimètre de longueur séparés par des distances de l'ordre du mètre. Pour l'observateur, la lumière est modulée avec superposition de trois fréquences dues à l'injection (quelques dizaines de Hertz) au mouvement orbital $(\sim 1 \mathrm{MHz})$ et à la cavité radiofréquence $(\sim 400 \mathrm{MHz})$.

Les anneaux de stockage ou anneaux de collision ont été conçus pour emmagasiner (dans le cas le plus simple) deux paquets de particules chargées de signe opposé $\left(\mathrm{e}^{-}, \mathrm{e}^{+}\right)$circulant dans l'anneau en sens inverse et entrant en collision périodiquement dans une des sections droites de la chambre à vide où des expériences de Physique des Hautes Energies sont implantées. Le mode de fonctionnement de ces machines est très différent de celui des synchrotrons. Les électrons (ou les positrons) sont injectés dans l'anneau à grande énergie par un accélérateur linéaire (ou par un autre synchrotron) à des faibles cadences (2-3 cycles/s), jusqu'à ce que l'intensité du courant d'électrons voulue soit atteinte. On fait alors croître le champ dans les aimants ainsi que la puissance de la cavité radiofréquence jusqu'à ce que les particules aient atteint l'énergie désirée. La cavité agissant comme un monochromateur concentre les électrons en un ou plusieurs paquets dont la dispersion en énergie est inférieure à $5 \times 10^{-4}$. Les particules tournent alors dans la machine pendant des temps très longs. La précision absolue sur la valeur de l'énergie est de l'ordre de $10^{-3}$.

Pour l'utilisation du rayonnement synchrotron, les anneaux de stockage présentent donc de nombreux avantages par rapport aux synchrotrons. La fluctuation de l'intensité émise dans un synchrotron se trouve éliminée avec un anneau, car les particules conservent rigoureusement la même énergie dans le temps. De plus la fluctuation de l'intensité émise dans un synchrotron due à la variation du nombre d'électrons par injection se trouve éliminée avec un anneau dans lequel le nombre d'électrons ne diminue que lentement par suite des collisions avec les molécules de gaz résiduel. Un anneau constitue donc une source sans bruit.

La structure temporelle du rayonnement est beaucoup plus simple que dans le cas du synchrotron. En effet, il y a dans un anneau un ou plusieurs paquets d'électrons d'une longueur typique de quelques dizaines de centimètres tournant à la vitesse de la lumière. Le rayonnement synchrotron est alors émis sous la forme de "pulses" d'une durée de l'ordre de la nanoseconde se répétant à la fréquence orbitale avec une précision qui est celle de l'oscillateur de quartz qui pilote le générateur RF.

La stabilité de l'orbite dans un anneau est très supérieure à ce qu'elle est dans un synchrotron. De plus le chargement de l'anneau est réalisé en une seule fois pour un grand laps de temps, et une fois les électrons accélérés à leur énergie maximum, il n'y a pas de rayonnement $\gamma$ émis autour de l'anneau, ce qui permet de travailler en permanence dans la salle expérimentale, une fois l'anneau chargé. Le contrôle à distance des expériences par télécommande, indispensable auprès d'un synchrotron, est inutile dans le cas d'un anneau.

4.2.3 Caractéristiques de l'anneau de stockage d'Orsay $A C O$ et du rayonnement qu'il émet. a) L'anneau. - ACO est un anneau de stockage à électrons et positrons en service à Orsay depuis 1966 [15]. Le rayon magnétique $\mathrm{Rm}$ de l'anneau est de $1,11 \mathrm{~m}$, la longueur totale de la trajectoire moyenne des électrons est de $22 \mathrm{~m}$. Le vide qui règne dans la chambre expérimentale est de l'ordre de $10^{-12} \mathrm{~Pa}$. La fréquence de rotation est de $13,618 \mathrm{MHz}$. L'énergie maximum qu'il est possible d'atteindre est de $540 \mathrm{MeV}$. L'énergie d'injection est ordinairement de $240 \mathrm{MeV}$. L'accélérateur linéaire d'Orsay de $1 \mathrm{GeV}$ est utilisé pour l'injection des électrons et des positrons. La ligne destinée à utiliser le rayonnement synchrotron sort tangentiellement à l'orbite dans l'un des aimants et voit 10 milliradians de la trajectoire. La description de l'ensemble des installations du laboratoire pour l'utilisation du rayonnement électromagnétique (L. U. R. E.), récemment créé pour rendre utilisable le rayonnement synchrotron d'ACO, a été présentée précédemment [16]. Lorsque seuls des électrons sont injectés dans la machine, le courant total maximum qu'il est possible d'atteindre à $540 \mathrm{MeV}$ est de plusieurs centaines de milliampères, répartis en un ou deux paquets d'électrons, mais il est actuellement limité à $100 \mathrm{~mA}$ pour éviter de détériorer les passages de courant des électrodes. Le rayonnement émis dans la ligne de lumière est pulsé : la durée de chaque pulse est de $1 \mathrm{~ns}$, l'intervalle entre chaque pulse étant de 73 ou $36,5 \mathrm{~ns}$, suivant que un ou deux paquets sont présents dans l'anneau. Avec un courant de $100 \mathrm{~mA}$, le vide mesuré est de $2 \times 10^{-12} \mathrm{~Pa}$ et la 
durée de vie moyenne du faisceau est d'environ 12 heures pour un paquet et 16 heures pour 2 paquets d'électrons. Cette durée de vie dépend de la densité des particules dans le faisceau et on peut la faire varier en agissant sur la forme et les dimensions de celui-ci. Quel que soit le courant présent, il diminue de façon extrêmement régulière et le bruit relatif de cette source de rayonnement est inférieur à $10^{-5}$.

Les largeurs à mi-hauteur des distributions gaussiennes de la densité électronique dans les plans horizontal et vertical pour un tel faisceau sont d'environ $1 \mathrm{~mm}$ pour un faisceau rond et respectivement de $1,5 \mathrm{~mm}$ et $0,3 \mathrm{~mm}$ pour un faisceau plat. La stabilité du faisceau dans la chambre à vide est meilleure que $\pm 20 \mu \mathrm{m}$. Cette source de lumière est donc également extrêmement stable dans l'espace.

b) Caractéristiques du rayonnement synchrotron émis par ACO [17]. - Puissance rayonnée. - La puissance totale rayonnée dans tout l'espace peut s'obtenir à partir de l'énergie $\Delta E$ perdue par un électron d'énergie $E$ pour une révolution (formule (7)). En unités pratiques, cette relation s'écrit :

$$
\Delta E=88,5(E)^{4} R_{\mathrm{m}}^{-1}
$$

pour $E$ exprimé en $\mathrm{GeV}$ et $R_{\mathrm{m}}$ en mètre. $\Delta E$ est exprimé en $\mathrm{keV}$. Pour ACO à $540 \mathrm{MeV}$, on obtient

$$
\Delta E=6,84 \mathrm{keV} \text {. }
$$

Dans le cas d'un anneau de stockage, cette expression est directement transposable au calcul de l'énergie rayonnée par le courant d'électrons. Il suffit en effet de multiplier la valeur pour un électron par le nombre d'électrons injectés dans l'anneau, à condition de prendre pour rayon $R$ le rayon magnétique de la trajectoire, qui est égal au rayon de courbure de la trajectoire dans les aimants, et de tenir compte du fait que le faisceau d'électrons n'émet de rayonnement que dans les parties courbées Comme les effets collectifs à l'intérieur du faisceau d'électrons sont négligeables, l'énergie rayonnée est simplement égale à la somme des énergies émises indépendamment par chaque électron.

Remarquons qu'il ne peut en être de même dans le cas d'un synchrotron qui répète un certain nombre de fois par seconde un cycle d'accélération des électrons à partir de l'énergie d'injection jusqu'à l'ênergie maximum; les calculs doivent alors tenir compte du fait que l'électron ne circule pas sur sa trajectoire pendant toute la durée du cycle avec l'énergie maximum.

Pour un courant maximum de $100 \mathrm{~mA}$ à $540 \mathrm{MeV}$, la puissance totale rayonnée dans tout l'espace et dans tout le spectre continu est ainsi de 685 watts pour ACO.

La puissance instantanée $P(\lambda)$ rayonnée par un électron à une longueur d'onde $\lambda$, par intervalle unité de longueur d'onde, dans tout l'espace, a pour expression :

$$
P(\lambda)=\frac{3^{5 / 2}}{16 \pi^{2}} \frac{e^{2} c}{R^{3}} \gamma^{7} G(y)
$$

avec $y=\lambda_{\mathrm{c}} / \lambda$

$$
\begin{aligned}
\lambda_{\mathrm{c}} & =\frac{4 \pi R}{3} y^{-3} \\
G(y) & =y^{3} \int_{y}^{\infty} K_{5 / 3}(x) \mathrm{d} x .
\end{aligned}
$$

$K_{5 / 3}=$ fonction de Bessel modifiée de seconde espèce d'ordre $5 / 3$

$G(y)$ est une fonction sans dimension dont la courbe représentative a été calculée point par point [14].

$\lambda_{c}$ peut encore s'écrire, en unités pratiques :

$$
\lambda_{\mathrm{cnm}}=0,559 R_{\mathrm{m}}\left(E_{\mathrm{GeV}}\right)^{-3} \text {. }
$$

$\lambda_{\mathrm{c}}$ est appelée longueur d'onde critique et sa valeur est une des caractéristiques de la courbe d'émission spectrale. Dans le cas d'ACO à l'énergie maximum de $540 \mathrm{MeV}, \lambda_{\mathrm{c}}=3,9 \mathrm{~nm}$ avec $\gamma^{-1}=0,94 \times 10^{-3}$.

La distribution spectrale du rayonnement émis par ACO à $540 \mathrm{MeV}$ et pour un courant de $100 \mathrm{~mA}$ est représentée figure 5, exprimée en nombre de

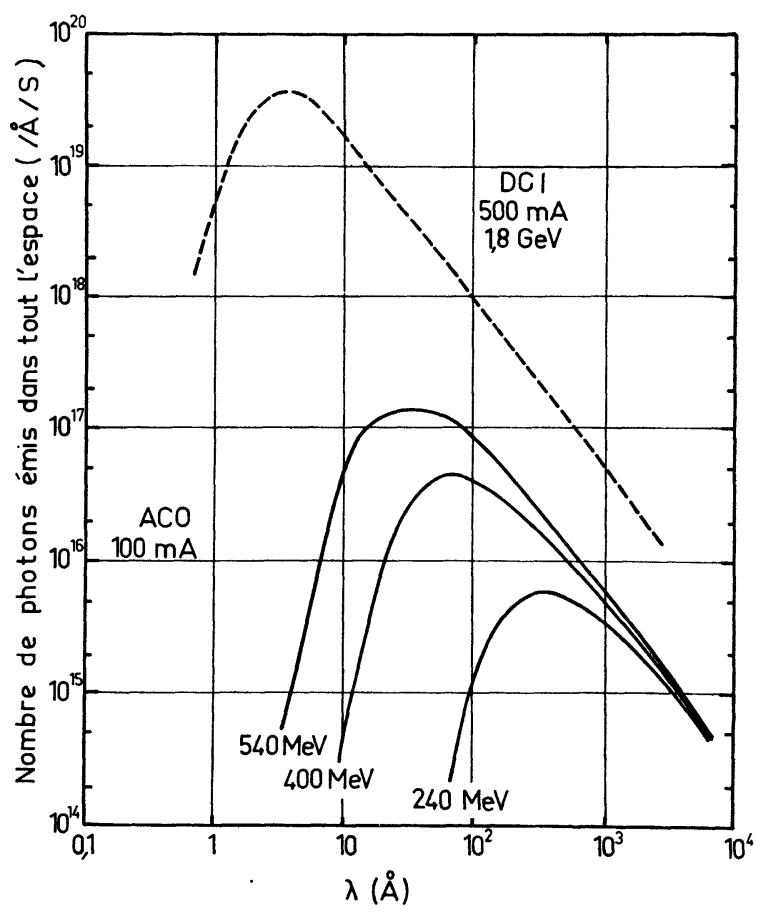

Fig. 5. - Nombre de photons émis dans tout l'espace par ACO en fonction de la longueur d'onde $\lambda$ pour différentes valeurs de l'énergie des électrons. On notera que l'intensité émise aux grandes longueurs d'onde est pratiquement indépendante de l'énergie des électrons. Le nombre de photons émis par DCI est indiqué pour l'énergie maximum des électrons. $1 \AA=0,1 \mathrm{~nm}$.

[Total number of photons emitted by ACO versus the wavelength for different electron energies. Note that the intensity for long wavelengths is almost independent of electron energy. We have reported the total number of photons emitted by DCI for the highest electron energy.] 
photons émis dans tout l'espace pour une bande passante de $0,1 \mathrm{~nm}$ et par seconde, pour plusieurs énergies des électrons. Ce nombre s'obtient directement en divisant la puissance $P(\lambda)$, relation (9), par l'énergie $h v$ correspondante. On notera que le spectre présente un maximum situé, à $540 \mathrm{MeV}$, aux environs de $3 \mathrm{~nm}\left(\lambda_{\mathrm{M}} \sim 0,76 \lambda_{\mathrm{c}}\right)$. L'intensité émise décroît très vite vers les courtes longueurs d'onde par rapport au maximum et diminue plus lentement vers les grandes longueurs d'onde. On remarquera également que l'intensité émise varie fortement avec l'énergie des électrons pour les courtes longueurs d'onde, inférieures à la longueur d'onde critique, alors que, vers les grandes longueurs d'onde, l'intensité devient pratiquement indépendante de l'énergie des électrons. Sur cette figure est également représentée la distribution spectrale du rayonnement émis par l'anneau de collisions DCI, actuellement en cours de mise au point à Orsay et dont le maximum se situe dans la région des rayons $\mathrm{X}$.

Le nombre de photons émis dans les deux états de polarisation se calcule exactement à partir des caractéristiques de la machine. L'expression générale de l'intensité émise à une longueur d'onde $\lambda$, en fonction de l'angle d'émission $\psi$, exprimée en nombre de photons par unité d'angle et par unité de longueur d'onde, peut s'écrire :

$$
\begin{gathered}
N(\psi, \lambda)=\frac{27}{32 \pi^{3}} \frac{e^{2} \lambda}{h R^{3}}\left[\frac{\lambda_{\mathrm{c}}}{\lambda}\right]^{4} \gamma^{12}\left[l_{2} \frac{1+\gamma^{2} \psi^{2}}{\gamma^{2}} K_{2 / 3}(x)+\right. \\
\left.+\frac{l_{3} \psi\left(1+\gamma^{2} \psi^{2}\right)^{1 / 2}}{\gamma} K_{1 / 3}(x)\right]^{2}
\end{gathered}
$$

avec

$$
x=\frac{\lambda_{c}}{2 \lambda}\left[1+(\gamma \psi)^{2}\right]^{3 / 2}
$$

$K_{1 / 3}, K_{2 / 3}=$ fonctions de Bessel modifiées de $2^{e}$ espèce d'ordre $1 / 3$ et $2 / 3$.

$K_{n / 3}(x)$ a pour expression, en fonction des fonctions de Bessel fractionnaires de première espèce d'ordre $n / 3$ :

$$
K_{n / 3}(x)=\frac{\pi}{2}\left[I_{-n / 3}(x)-I_{n / 3}(x)\right] / \sin \left(n \frac{\pi}{3}\right)
$$

ou sous forme intégrale :

$$
K_{n / 3}(x)=\int_{0}^{\infty} \exp (-x \cosh t) \cosh \frac{n t}{3} \mathrm{~d} t
$$

La composante $\sigma$ de polarisation linéaire, polarisée parallèlement au plan de l'orbite, s'obtient en faisant $l_{2}=1$ et $l_{3}=0$ dans la formule (13). Elle s'écrit donc :

$N_{\|}(\lambda, \psi)=\frac{27}{32 \pi^{3}} \frac{e^{2} \lambda}{h R^{3}}\left[\frac{\lambda_{\mathrm{c}}}{\lambda}\right]^{4} \gamma^{8}\left[1+(\gamma \psi)^{2}\right]^{2} K_{2 / 3}^{2}(x)$.
La composante perpendiculaire $\pi$ s'obtient en faisant $l_{2}=0$ et $l_{3}=1$ dans la formule (13) qui s'écrit alors :

$$
\begin{array}{r}
N_{\perp}(\lambda, \psi)=\frac{27}{32 \pi^{3}} \frac{e^{2} \lambda}{h R^{3}}\left[\frac{\lambda_{\mathrm{c}}}{\lambda}\right]^{4} \gamma^{8}\left[1+(\gamma \psi)^{2}\right] \\
(\gamma \psi)^{2} K_{1 / 3}^{2}(x) .
\end{array}
$$

La vibration résultante étant elliptique, elle peut être décomposée en deux vibrations circulaires l'une droite, l'autre gauche.

L'expression de ces composantes s'obtient en faisant

$$
l_{2}=l_{3}=\frac{1}{\sqrt{2}}
$$

dans la formule (13) pour la composante de polarisation circulaire droite et

$$
l_{2}=-l_{3}=\frac{1}{\sqrt{2}}
$$

pour la composante de polarisation circulaire gauche, si bien qu'on peut les exprimer de la façon suivante en fonction des composantes $\sigma$ et $\pi$ :

$$
\begin{aligned}
& N_{\mathrm{D}}=\frac{1}{2}\left[N_{\|}+N_{\perp}+2\left(N_{\|} \cdot N_{\perp}\right)^{1 / 2}\right] \\
& N_{\mathrm{G}}=\frac{1}{2}\left[N_{\|}+N_{\perp}-2\left(N_{\|} \cdot N_{\perp}\right)^{1 / 2}\right] .
\end{aligned}
$$

Ces expressions sont valables pour une direction d'émission située au-dessus du plan de l'orbite. Pour la direction symétrique située au-dessous, les formules pour les composantes droite et gauche doivent être inversées.

La figure 6 représente, à titre d'exemple, la variation, en fonction de $\psi$, de l'intensité relative des composantes parallèle et perpendiculaire émises par ACO pour le domaine de longueur d'onde compris entre 10 et $100 \mathrm{~nm}$. La composante parallèle a toujours une intensité maximum pour $\psi=0$, c'est-à-dire, dans le plan de l'orbite, le long de la tangente à ce.le-ci. Elle décroît plus ou moins vite lorsque l'angle $\psi$ augmente. La composante perpendiculaire a toujours une intensité nulle à $\psi=0$, et présente 2 maximums symétriques par rapport à la tangente à l'orbite, qui se déplacent vers les grandes valeurs de $\psi$ lorsque la longueur d'onde augmente et dont l'intensité varie beaucoup avec la longueur d'onde : rapportée à l'intensité de la composante parallèle pour $\psi=0$, elle est de quelques \% pour les courtes longueurs d'onde par rapport à la longueur d'onde critique ; mais elle atteint 35 à $40 \%$ aux grandes longueurs d'onde. Après le maximum, l'intensité de la composante perpendiculaire décroît, mais le rapport entre les deux composantes tend vers 1 pour les angles d'élévation plus élevés, la composante parallèle restant cependant toujours plus intense que la composante perpendiculaire.

La connaissance de la distribution angulaire du rayonnement permet de prévoir le nombre de photons 


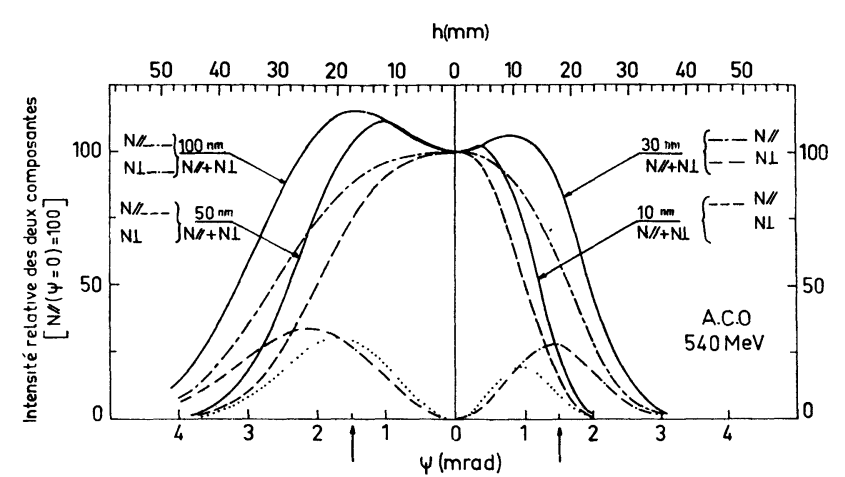

FIg. 6. - Intensité relative des composantes parallèle et perpendiculaire et intensité totale en fonction de l'angle d'émission $\psi$ entre 10 et $100 \mathrm{~nm}$ pour ACO à $540 \mathrm{MeV} . N_{\|}(\psi=0)=100$. La moitié seulement de la courbe est tracée pour chaque longueur d'onde, le rayonnement étant émis symétriquement par rapport au plan de l'orbite; $h$ est la hauteur de la fente nécessaire pour recevoir, à $12 \mathrm{~m}$, tout le rayonnement émis dans le demi-cône d'angle au sommet $\psi$ correspondant. L'ouverture du faisceau utilisable actuellement à LURE $(3 \mathrm{mrad})$ est indiquée par deux flèches. On notera que, lorsque la longueur d'onde augmente, les maxima de l'intensité totale émise s'éloignent symétriquement du plan de l'orbite. Dans cette figure les courbes donnent les valeurs correspondant à un angle d'émission $\psi$ déterminé, qui ne sont donc pas intégrées par rapport à $\psi$, à la différence de la figure 4 .

[Relative intensity of the parallel and perpendicular components and total intensity versus $\psi$ between 100 and $1000 \AA$ for ACO at $540 \mathrm{MeV} . N_{\|}(\psi=0)=100$. Since the radiation is symmetric about the orbital plane, we have only drawn half of the curve for each wavelength. $h$ is the slit height necessary to receive all the radiation emitted in the angle $\psi$ from 12 meters. The available aperture for the ACO is indicated by two arrows. As the wavelength increases the total intensity maxima move away symetrically from the orbital plane. The curves show the values corresponding to a given $\psi$.]

que l'on peut espérer recevoir effectivement dans une configuration géométrique déterminée, par exemple sur la fente d'entrée d'un monochromateur. Ce nombre utile de photons est la caractéristique la plus importante à connaître pour les expériences à réaliser. Sa variation est représentée figure 7 pour plusieurs valeurs d'ouverture angulaire du faisceau et pour deux énergies différentes des électrons (400 et $540 \mathrm{MeV}$ ).

Le taux de polarisation linéaire du rayonnement est défini par la relation :

$$
P=\frac{N_{\|}-N_{\perp}}{N_{\|}+N_{\perp}}
$$

et le taux de polarisation circulaire par :

$$
\tau_{\mathrm{c}}=\frac{N_{\mathrm{D}}-N_{\mathrm{G}}}{N_{\mathrm{D}}+N_{\mathrm{G}}} .
$$

Les figures 8 et 9 montrent les variations de ces taux en fonction de l'angle $\psi$ pour diverses longueurs d'onde.

Mentionnons que les caractérisiques détaillées des flux de photons émis et disponibles aux diverses lon-

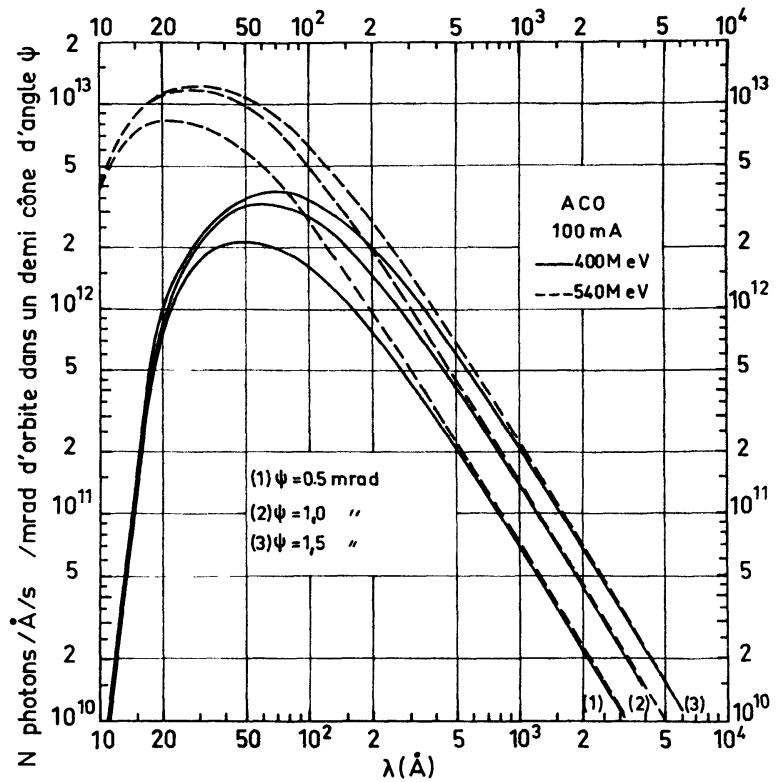

Fig. 7. - Variation, en fonction de la longueur d'onde, du flux de photons émis par ACO, par angstrom, par seconde, par milliradian d'orbite, dans le demi-plan vertical délimité par le demicône d'angle au sommet $\psi$, pour un courant de $100 \mathrm{~mA}$, à 540 et $400 \mathrm{MeV}$. Les caractéristiques du rayonnement sont indiquées ainsi, car les montages expérimentaux sur ACO divisent le faisceau dans le plan vertical, certains utilisant le rayonnement émis au-dessus du plan de l'orbite et d'autres le rayonnement émis en dessous de ce plan.

[Variation of the photon flux per $\AA$, per second for one milliradian in the half plane defined by $\psi$ for $100 \mathrm{~mA}, 540 \mathrm{MeV}$ and $400 \mathrm{MeV}$.]

gueurs d'onde ont fait l'objet de calculs systématiques dont les résultats ont été publiés dans un précédent rapport [17].

4.2.4 Photométrie avec le rayonnement synchrotron. - Les formules rappelées ci-dessus, qui donnent les flux de photons dans des conditions déter-

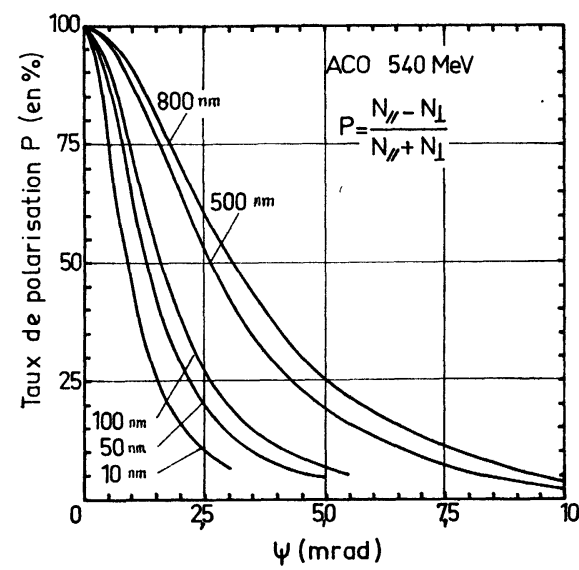

Fig. 8. - Taux de polarisation linéaire du rayonnement émis par ACO à $540 \mathrm{MeV}$ entre 10 et $800 \mathrm{~nm}$ en fonction de l'angle d'émission.

[Linear polarization rate at $540 \mathrm{MeV}$ between 100 and $8000 \AA$ versus $\psi$.] 


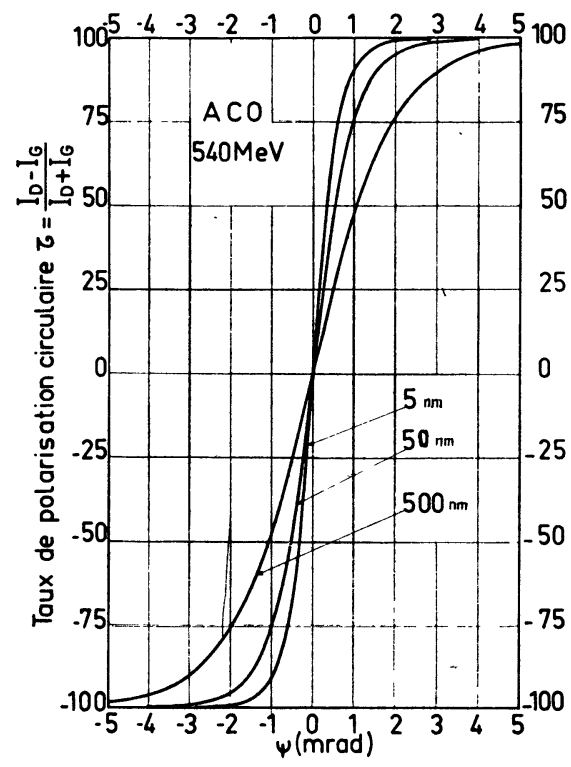

Fig. 9. - Taux de polarisation circulaire du rayonnement émis par ACO, à $540 \mathrm{MeV}$, à 5,50 et $500 \mathrm{~nm}$ en fonction de l'angle d'émission.

[Circular polarization rate at $540 \mathrm{MeV}$ versus $\psi$ for 50,500 , $5000 \AA$.

minées, ont été vérifiées expérimentalement sur le synchrotron à électrons de $300 \mathrm{MeV}$ de l'Université de Cornell aux Etats-Unis [18] puis sur les synchrotrons de $280 \mathrm{MeV}$ et $660 \mathrm{MeV}$ de l'Institut Lebedev de l'Académie des Sciences de l'U. R. S. S. [19]. L'existence d'une polarisation elliptique a ensuite été confirmée expérimentalement sur le rayonnement émis par le synchrotron de Cornell [20] et par celui de $180 \mathrm{MeV}$ du National Bureau of Standards de Washington [21]. Soulignons cependant que, dû aux variations spatiales du faisceau d'électrons dans les synchrotrons, le taux de polarisation linéaire n'a jamais été trouvé égal à $100 \%$ dans le plan même de l'orbite pour ce type de machines. Dans tous les cas la composante perpendiculaire n'était jamais nulle dans le plan de l'orbite. Les synchrotrons se sont révélés cependant comme des sources étalons de rayonnement extrêmement précieuses dans l'ultraviolet extrême et ont déjà fait l'objet de nombreuses utilisations de ce genre [22, 23, 24].

La première vérification expérimentale effectuée à LURE, des résultats de la théorie pour un anneau de stockage tel qu'ACO, ont fourni un accord excellent entre les flux calculés et les flux mesurés. De plus la composante perpendiculaire a été effectivement trouvée nulle, dans le plan de l'orbite, comme on peut le voir figure 10 où sont représentées les variations expérimentale et théorique du taux de polarisation circulaire en fonction de l'angle d'émission qui montre un accord remarquable entre la théorie et l'expérience. L'ensemble de l'expérience doit être décrit par ailleurs [25].

Ces expériences préliminaires ont démontré pleine-

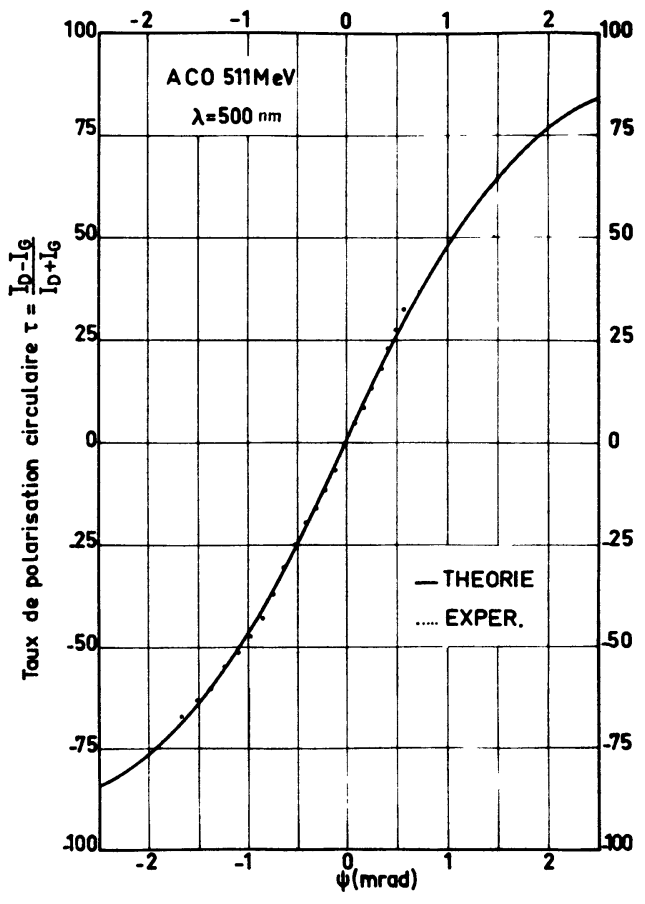

FIG. 10. - Comparaison du taux de polarisation circulaire mesurée en fonction de l'angle d'émission $\psi$ (points) et calculée à partir des formules théoriques (courbe en trait plein).

[Comparison of the theorical circular polarization rate (full curve) with experiment (dots).]

ment les larges possibilités offertes par un anneau de stockage tel qu'ACO et deux expériences de photométrie [16] l'une destinée à l'étalonnage d'un spectrographe UV qui doit être embarqué dans un satellite, l'autre conçue pour vérifier les caractéristiques d'un étalon de température constitué d'un arc à hydrogène, sont actuellement en cours, en vue d'utiliser le rayonnement synchrotron émis par ACO comme un étalon de rayonnement.

4.3 Rayonnement Cerenkov [26]. - Le rayonnement Cerenkov se produit lorsque des particules de grande énergie traversent un matériau à une vitesse $v$ supérieure à la vitesse de la lumière $v=c / n$ dans ce matériau, $n$ étant l'indice de réfraction. Un large spectre de lumière polarisée est émis suivant un cône faisant un angle $\theta_{\mathrm{c}}$ avec le faisceau de particules, tel que $\cos \theta_{c}=c / n v$. Le champ électrique du rayonnement est perpendiculaire à la surface du cône et le champ magnétique est tangentiel. L'énergie minimum pour que l'émission Cerenkov se produise, par exemple dans l'hélium, est de $55 \mathrm{MeV}$. On définit une énergie optimum pour laquelle les pertes par ionisation et rayonnement de freinage sont minima ; elle est de $260 \mathrm{MeV}$ dans le cas de l'hélium. Ce phénomène ne peut évidemment se produire que si $n$ est plus grand que un. Le matériau doit être également transparent dans le domaine spectral d'émission. Enfin, il faut qu'il supporte un faisceau d'électrons relativistes sans dommage. Le tableau I donne une 
liste de corps et leur longueur d'onde de coupure $\lambda_{c}$ pour laquelle l'indice devient inférieur à l'unité ainsi que leur indice de réfraction à $250 \mathrm{~nm}$.

\section{TABLEAU III}

$\begin{array}{lcl} & \lambda_{\mathrm{c} \mathrm{nm}} & n_{(2500 \mathrm{~nm})} \\ \text { Hélium } & \overline{58,4} & 1,000036 \\ \text { Néon } & 73,6 & 1,000069 \\ \text { Argon } & 104,8 & 1,0003 \\ \mathrm{LiF} & 105 & 1,418 \\ \mathrm{CaF}_{2} & 135 & 1,4673 \\ \text { Saphir } & 142,5 & 1,845 \\ \text { Silice } & 165 & 1,5074\end{array}$

La puissance émise par $\AA$ et par électron est calculable, d'où l'utilisation du rayonnement Cerenkov comme source étalon. Elle est égale à :

$$
\frac{\mathrm{d} P}{\mathrm{~d} \lambda}=2,8 \times 10^{-1} \cdot \frac{\sin ^{2} \theta_{\mathrm{c}}}{\lambda^{3}}
$$

où $P$ est mesurée en watts et $\lambda$ en $\mathrm{nm}$. La lumière est émise avec très peu de divergence; l'angle du cône d'émission pour de l'hélium à $65 \mathrm{~nm}$ est de $2,8 \times 10^{-2}$ rd. A titre de comparaison la puissance spectrale émise par l'électron est de 100 à 1000 fois supérieure à celle rayonnée par un électron dans un synchrotron de $180 \mathrm{MeV}$.

4.4 LA CROIX D'ÉTALONNAGe [28]. - Une nouvelle technique permet d'étalonner des appareils de transfert dans l'ultraviolet (sources ou détecteurs). Cette méthode est basée sur la comparaison entre un flux ultraviolet de faible bande passante provenant d'un monochromateur et le flux total provenant d'un corps noir ayant une température d'environ $500 \mathrm{~K}$. Une analyse détaillée des erreurs indique que les incertitudes sont de l'ordre de $20 \%$.
5. Etalons de transfert. - Seule la méthode du paragraphe 3.2 peut être employée in situ dans une expérience. En général, pour l'étalonnage d'un appareil ou d'une source, on ne peut pas utiliser, pour des raisons pratiques, l'étalon primaire. Il faut donc assurer la liaison entre l'étalon primaire et l'appareil à étalonner. On dispose pour cela des sources dites de transfert calibrées par rapport à l'étalon primaire ou étalons secondaires. Pour constituer des étalons secondaires, il est nécessaire que ces sources aient des qualités métrologiques comparables à l'étalon primaire et surtout qu'elles soient stables et reproductibles. De plus, leur emploi doit être pratique et sans ambiguité. Dans le domaine visible la lampe à ruban de tungstène constitue une excellente source de transfert. En dessous de $300 \mathrm{~nm}$ seules les lampes à décharges de type lampes à deutérium, ou lampes à mercure sont utilisées assez couramment. La lampe à deutérium donne un spectre continu entre $165 \mathrm{~nm}$ $300 \mathrm{~nm}$; le spectre de la lampe à mercure est continu avec ou sans raies suivant les réalisations.

Pour descendre en dessous de $165 \mathrm{~nm}$ un mini arc est actuellement à l'étude au National Bureau of Standard [30]. Il fonctionne sous pression atmosphérique d'argon, ne nécessite qu'un refroidissement par air et une alimentation transportable ; un type d'arc similaire est à l'étude à l'Institut National de Métrologie. D'autre part, Guenther ct al. [31] étudie une source pulsée qui rayonne comme un corps noir à $12000 \mathrm{~K}$.

6. Conclusion. - L'étalonnage ou la mesure d'énergie rayonnante dans le domaine ultraviolet est à l'heure actuelle un problème assez difficile. Le choix d'une méthode dépend de nombreux paramètres (domaine de longueur d'onde, ordre de grandeur de l'énergie à mesurer, application, etc...) et l'utilisateur doit souvent adapter cette méthode à ses propres besoins. Un effort important est actuellement entrepris par l'Institut National de Métrologie pour fournir des étalons de transfert étalonnés par ses soins.

\section{Bibliographie}

[1] Cristofori, F., Fenici, P., Frigerio, G. E., Molho, N., Sona, P. G., Phys. Lett. 6 (1963) 171.

[2] Bethe, H., Ann. der Phys. (1930), 325 ou Handbuch der Physik (Springer, Berlin), 1933, 2411, 273.

[3] Inokuti, M., Rev. Mod. Phys. 43 (1971) 297.

[4] Van RaAn, A. F. J., Physica 65 (1973) 566.

[5] Griffen, W. G., McWhiter, R. W. P., Conference on optical instruments and techniques. Ed. K. J. Habell (Chapman and Hœl, London) 1962.

[6] Mumma, M. J., J. Opt. Soc. Am. 62, 12 (1972) 1459.

[7] Herzberg, G., Spectra of diatomic molecules (Van Nostrand Reinhol Compagny).

[8] Boldt, G., Space. Sc. Rev. 11 (1970) 728 ; Key, P.-J., Preston, R. C., Appl. Opt. (à paraître).

[9] Lochte-Holtgreven, W., Plasma diagnostics (North Holland) ou

GrIEM, H., Plasma Spectroscopy (McGraw-Hill).
[10] Stuck, D., Wende, B., J. Opt. Soc. Am. 62 (1972) 1. Key, P.-J., Preston, R. C., Nature (en voie de publication).

[11] RoberTs, J. R., VoIGT, P. A., J. Res. Nat. Bus. Stand. 75A 4 (1971) 291.

[12] MAeCKer, H., Z. Naturforsch. 11 (1956) 457.

MaeCKer, H., Steinberger, Urban, $Z$. Angew Phys. 15 (1963) 440.

[13] Оtт, W. R., Fieffe-Prevost, P., Wiese, W. L., Appl. Opt. 12 (1973) 1618.

[14] La théorie du rayonnement synchrotron a été élaborée presque simultanément par :

SCHWINGER, J., Phys. Rev. 75 (1949) 1912 et

Ivanenko, D. et Sokolov, A. A., Dokl. Akad. Nauk. (URSS) 59 (1948) 1551. On en trouvera un exposé complet dans les ouvrages suivants :

Sokolov, A. A., Ternov, I. M., Synchrotron Radiation (Pergamon Press) 1968. 
Cauchois, Y. et Heno, Y., Cheminement des particules chargées (Gauthier-Villars, Paris) 1964.

Godwin, R. P., Tracts in Modern Physics, vol. 51 (SpringerVerlag, Berlin) 1969.

Coduing, K., Rep. Progr. Phys. 36 (1973) 541.

[15] Comptes rendus des $4^{\mathrm{e}}, 5^{\mathrm{e}}$ et $6^{\mathrm{e}}$ " International Conference on High Energy Accelerators » qui se sont tenues respectivement à : Dubna (1963), Frascati (1965) et Cambridge (1967).

[16] Dagneaux, D., Depautex, C., Dhez, P., Durup, J., Farge, Y., Fourme, R., GuYon, P. M., JaEgle, P., Leach, S., Lopez-Delgado, R., Morel, G., PiNChauX, R., Thiry, P., Vermeil, C. et WuIlleumier, F., Annl Phys. 9 (1975) 9.

[17] WUILleumieR, F., Rapport LURE 74/03 (Laboratoire commun au C. N. R. S. et à l'Université Paris-Sud) Orsay (1974).

[18] Tomboulian, D. H. et Hartman, P. L., Phys. Rev. 102 (1956) 1923.
[19] Sokolov, A. A. et Ternov, I. M., Synchrotron Radiation (Pergamon Press) 1968, p. 179 et suivantes.

[20] Joos, P., Phys. Rev. Lett. 4 (1960) 558.

[21] Codling, K. et Madden, R. P., J. Appl. Phys. 36 (1964) 380.

[22] PITz, E., Appl. Opt. 8 (1969) 255.

[23] Key, P. J., Metrologia 6 (1970) 97.

[24] Rusbuldt, D. et ThImM, K., Nucl. Instrum. Meth. 116 (1974) 125.

[25] Fieffe-Prevost, P., Bastie, J., Farge, Y. et WuilleuMIER, F., à paraître.

[26] Piestrüp, M. A., Pantell, R. H., Puthoff, H. E., RothBART, G. B., J. Appl. Phys. 44 (1973) 11.

[27] OtT, W. R., Behringer, K., Gieres, G., Appl. Opt. 14 (1975) 2121.

[28] MARETTE, G., Appl. Opt. 15 (1976) 440.

[29] FIefFe-Prevost, P., Physics of Ionized Gases, SPIG 76.

[30] Bridges, J. M., Otт, W. R., Appl. Opt. 16 (1977) 367.

[31] GUENTHER K., et al., Private communication, GUENTHER, K., RADTKE, R., J. Phys. E, Scientific Instrument, 8 (1975). 